\title{
Funktionelle Dickdarmchirurgie
}

\author{
Asad Kutup $^{\mathrm{a}} \quad$ Jutta Keller $^{\mathrm{b}} \quad$ Peter Layer $^{\mathrm{b}} \quad$ Jacob R. Izbicki ${ }^{\mathrm{a}}$ \\ ${ }^{a}$ Klinik und Poliklinik für Allgemein-, Viszeral- und Thoraxchirurgie, Universitätsklinikum Hamburg-Eppendorf, \\ ${ }^{\mathrm{b}}$ Medizinische Klinik, Israelitisches Krankenhaus, Lehrkrankenhaus der Universität Hamburg, Hamburg, Deutschland
}

Funktionelle Darmerkrankungen werden aus medizinischer Sicht aufgrund der normalen Lebenserwartung häufig als harmlos angesehen, können aber mit unterschiedlich ausgeprägten Beeinträchtigungen von Alltagsfunktionen und einer erheblichen Einschränkung der Lebensqualität einhergehen. Die Ursache solcher Störungen ist bislang unklar, auch wenn die international etablierten Diagnosekriterien nach Rom III darauf hinweisen, dass die zukünftige Forschung biochemische oder strukturelle Störungen bei funktionellen Darmstörungen nachweisen wird - allerdings nicht mit aktuell verfügbaren Routinemethoden.

Funktionelle gastrointestinale Erkrankungen gehen vielfach mit Stuhlgangsveränderungen einher. Die chronische Obstipation ist eine der möglichen Ausprägungsformen und kann die Patienten ganz besonders belasten. Moderne Definitionen der chronischen Obstipation richten sich auch deshalb nicht allein nach «objektiven» Parametern wie einer verminderten Stuhlfrequenz, sondern berücksichtigen, dass eine Obstipation vorwiegend durch subjektiv inadäquate, unbefriedigende Stuhlentleerungen definiert wird.

Die optimale Behandlung von Patienten mit chronischer Obstipation stellt wegen der vielfältigen Ursachen und klinischen Ausprägungsformen sowie einer Vielzahl von konservativen und operativen Therapieoptionen weiterhin eine Herausforderung dar.

Die allermeisten Patienten mit chronischer Obstipation profitieren ausreichend von einer medikamentös-konservativen Therapie. Es gibt aber durchaus Situationen, in denen solche Maßnahmen nicht genügen, sondern funktionelle Dickdarmchirurgie angebracht ist. Dies kann Patienten mit besonders stark ausgeprägten Motilitätsstörungen betreffen oder solche, bei denen anatomisch-morphologische Veränderungen die Symptomatik verursachen oder zumindest wesentlich erschweren. Hierbei hängt der Therapieerfolg in hohem Maße von der sorgfältigen Patientenselektion, der Indikationsstellung und auch der Wahl der chirurgischen Maßnahme ab, die somit eine besondere Herausforderung darstellen.

Bislang besteht bei vielen schwer betroffenen Patienten ein erheblicher Leidensdruck, während Ärzte aller Disziplinen, die sich mit dieser Problematik befassen, in Bezug auf diagnostische und therapeutische Maßnahmen oft unsicher und frustriert sind.

Vor diesem Hintergrund soll das aktuelle Schwerpunktheft dem Leser eine Orientierung bieten und die relevanten Informationen zu Ätiologie, Diagnostik und Therapiemöglichkeiten bei chronischer Obstipation vermitteln. Hierbei wird zunächst eine moderne Systematik und Diagnostik der chronischen Obstipation aufgezeigt. Die wichtige Unterscheidung in Kolontransitstörung und rektale Entleerungsstörung wird dabei in Beiträgen von Frieling [1] und Fürst et al. [2] berücksichtigt. Weitere Kapitel von Keller und Layer [3] sowie Pehl [4] stellen die medikamentös-konservativen Therapieoptionen bei funktioneller Obstipation, Reizdarmsyndrom mit Obstipation, «slow transit constipation» und sekundären Obstipationsformen dar.

Diese Kapitel dienen als Grundlage für die abschließenden Ausführungen zur funktionellen Dickdarmchirurgie bei Kolontransitstörungen durch Zehler und Kutup [5] sowie bei rektalen Entleerungsstörungen im Beitrag von Schwandner [6], die den eigentlichen Themenschwerpunkt des vorliegenden Heftes der VISZERALMEDIZIN detailliert darstellen.

\section{Literatur}

Frieling T: Systematik und Diagnostik der funktionellen Obstipation und Slow Transit Constipation. Viszeralmedizin 2012;28:231-236.

2 Fürst A, Keßler A, Liebig-Hörl G: Systematik und Diagnostik des obstruktiven Defäkationssyndroms. Viszeralmedizin 2012;28:237-242.
Keller J, Layer P: Medikamentös-konservative Therapieoptionen der Obstipation bei primären Funktionsstörungen des Kolons - vom Reizdarmsyndrom bis zur «Slow Transit Constipation». Viszeralmedizin 2012; 28:243-249.

4 Pehl C: Sekundäre Form der Obstipation aufgrund von medikamentösen Nebenwirkungen und endokrinen, neurologischen und psychiatrischen Erkrankungen medikamentös-konservative Therapieoptionen. Viszeralmedizin 2012;28:250-254.
5 Zehler O, Kutup A: Indikationen und Therapieoptionen aus chirurgischer Sicht bei der Kolontransitstörung. Viszeralmedizin 2012;28:255-259.

6 Schwandner O: Indikationen und chirurgische Therapieoptionen beim obstruktiven Defäkationssyndrom. Viszeralmedizin 2012;28:260-266.

\section{KARGER}

Fax +497614520714

Information@Karger.de

www.karger.com (c) 2012 S. Karger GmbH, Freiburg

$1662-6664 / 12 / 0284-0230 \$ 38.00 / 0$

Accessible online at:

www.karger.com/vim
Dr. med. Asad Kutup

Klinik und Poliklinik für Alloemein-, Viszeral- und Thoraxchirurgie

Universitätsklinikum Hamburg-Eppendorf

Martinistraße 52, 20246 Hamburg, Deutschland

Tel. +49 40 7410-58369, Fax -46756

kutup@uke.de 